\title{
Intermittent Hypoxia during Development Induces Long-Term Alterations in Spatial Working Memory, Monoamines, and Dendritic Branching in Rat Frontal Cortex
}

\author{
LEILA KHEIRANDISH, DAVID GOZAL, JEAN-MARC PEQUIGNOT, \\ JACQUELINE PEQUIGNOT, AND BARRY W. ROW
}

\begin{abstract}
Department of Pediatrics [L.K., D.G., B.W.R.], Kosair Children's Hospital Research Institute, Department of Pharmacology and Toxicology [D.G.], University of Louisville, Louisville, Kentucky 40202, Physiologie des Regulations Energetiques, Cellulaires et Moleculaires [J.-M.P., J.P.], CNRS, Université Claude

Bernard Lyon I, Campus de la Dowa, 69622 Villeurbanne, France
\end{abstract}

\begin{abstract}
ABS
Exposure to intermittent hypoxia (IH), such as occurs in
sleep-disordered breathing, is associated with increased apopto-
sis in vulnerable brain regions as well as with spatial reference
memory deficits in adult and developing rats. The latter are more
susceptible to IH, suggesting that early exposure to IH may have
long-term consequences. Rats were exposed to 14 d of room air
(RA) or IH starting at postnatal d 10 . Working memory was then
assessed in the water maze at 4 mo of age using a delayed
matching to place task in which the rats were required to locate
a submerged platform hidden in a novel location on the first trial
(T1 or acquisition trial), and then remember that position after a
delay (T2 or test trial). Mean escape latencies and swim distances
were derived and the savings (T1-T2) were used as a measure of
working memory. Male but not female rats exposed to IH
showed working memory deficits at both a 10- and 120-min
delay (for both latency and pathlength). Additionally, Sholl
analysis of Golgi-stained neurons revealed decreased dendritic
branching in the frontal cortex, but not the hippocampus, of male
\end{abstract}
OSA is a highly prevalent condition that affects $2-3 \%$ of all children (1-3). It is characterized by the repetitive development of either complete or partial upper airway occlusion that leads to periodic hypoxemia and hypercapnia and to recurring arousals. In recent years, it has become apparent that OSA imposes substantial neurobehavioral morbidity (4-6), particularly of functions pertaining to the PFC (7), and that such alterations in attention, executive, and intellectual function

Received August 17, 2004; accepted January 19, 2005.

Correspondence: David Gozal, M.D., Kosair Children's Hospital Research Institute, Department of Pediatrics, 570 South Preston St., Suite 321, Louisville, KY 40202; e-mail: david.gozal@louisville.edu

Supported by National Institutes of Health grants HL69932, HL63912, and P50 HL60296 (D.G.) and HD42395 (B.W.R.), and The Commonwealth of Kentucky Research Challenge Trust Fund.

DOI: 10.1203/01.pdr.0000176915.19287.e2 rats exposed to IH. Norepinephrine concentrations, dopamine turnover, and tyrosine hydroxylase activity were increased similarly in males and females. However, increased dopamine concentrations were present only in the frontal cortex of female rats. In conclusion, exposure to $\mathrm{IH}$ during a critical developmental period is associated with long-term alterations in frontal cortical dopaminergic pathways that may underlie gender differences in neurobehavioral deficits. (Pediatr Res 58: 594-599, 2005)
Cx, somatic cortex
DA, dopamine
IH, intermittent hypoxia
OSA, obstructive sleep apnea
PFC, pre-frontal cortex
RA, room air
TH, tyrosine hydroxylase

Abbreviations may not be completely reversible (8). The recent development of a rodent model, whereby IH is applied during sleep, has allowed for improved delineation of some of the potential mechanisms underlying the morbid consequences of OSA $(8-17)$. Furthermore, a unique period of neuronal susceptibility emerged, such that developing rats exposed to IH displayed reduced apoptosis during the immediate postnatal period but markedly enhanced neuronal cell loss between 10 and $25 \mathrm{~d}$ of age, compared with adult animals (18). Furthermore, exposure to IH during this period was associated with marked reductions in the ability to acquire a spatial task in the water maze and with locomotor hyperactivity in males but not in females (9). The potential irreversibility of the IH-induced effects was further suggested by Decker and colleagues (19), who reported altered dopaminergic transmission in rats exposed perinatally 
to a short period of $\mathrm{IH}$. We therefore hypothesized that longterm consequences of $\mathrm{IH}$, when occurring during a unique window of developmental brain susceptibility, may particularly affect male animals in prefrontal cortex related functions such as spatial working memory.

\section{METHODS}

Six time-pregnant Sprague-Dawley rats were purchased from a commercial breeder (Charles River Laboratories, Wilmington, MA), and delivery times were recorded. The experimental protocols were approved by the Institutiona Animal Use and Care Committee and are in close agreement with the National Institutes of Health guide for the care and use of laboratory animals. All efforts were made to minimize animal suffering, to reduce the number of animals used, and to use alternatives to in vivo techniques.

Chronic Intermittent Hypoxia Exposures

From d 10 to d 30 of life, animals were maintained with their dams in four identical commercially designed chambers $(30$ in $\times 20$ in $\times 20$ in; Oxycycler model A44XO, BioSpherix, Redfield, NY) that can accommodate three litters each, and were operated under a 12-h light-dark cycle (0600-1800 h). Gas was circulated around each of the chambers, attached tubing and other units at 60 $\mathrm{L} \cdot \mathrm{min}^{-1}$ (i.e. one complete change per $10 \mathrm{~s}$ ). The $\mathrm{O}_{2}$ concentration was continuously measured by an $\mathrm{O}_{2}$ analyzer and was changed throughout the $12 \mathrm{~h}$ of light time by a computerized system controlling the gas valve outlets, such that the moment-to-moment desired oxygen concentration of the chamber was programmed and adjusted automatically. The $\mathrm{IH}$ exposure profile consisted of 90 -s periods of $10 \% \mathrm{O}_{2}$ alternating with $21 \% \mathrm{O}_{2}$. Deviations from the desired concentration were met by addition of $\mathrm{N}_{2}$ or $\mathrm{O}_{2}$ through solenoid valves. This profile is associated with maximal reductions in oxygen blood tension with values ranging between 40 and $48 \mathrm{~mm} \mathrm{Hg}$ in adult rats. Approximately 20 such cycles are administered per hour of exposure, such that 240 cycles occur per day. For the remaining $12 \mathrm{~h}$ of nighttime, oxygen concentrations were kept at $21 \% \mathrm{O}_{2}$. Control litters (RA) were exposed to $21 \% \mathrm{O}_{2}$ throughout the $20-\mathrm{c}$ exposure period. Ambient $\mathrm{CO}_{2}$ in the chamber was periodically monitored and maintained at $<0.01 \%$ by adjusting overall chamber basal ventilation. The gas was also circulated through a molecular sieve (Type 3A, Fisons, Loughborough, UK) so as to remove ammonia. Humidity was measured and maintained at $40-50 \%$ by circulating the gas through a freezer and silica gel. Ambient temperature was kept at $22-24^{\circ} \mathrm{C}$. Upon completion of the exposures, animals were returned to the vivarial facility, weaned, and kept in cages of either three males or three females per cage until 4-5 mo of age, at which point in time they were studied.

Behavioral testing. The Morris water maze consisted of a circular pool, $1.8 \mathrm{~m}$ in diameter and $0.6 \mathrm{~m}$ in height, filled to a level of $35 \mathrm{~cm}$ with water maintained at a temperature of $27^{\circ} \mathrm{C}$. Pool water was made opaque by addition of $150 \mathrm{~mL}$ of nontoxic white tempera paint. A Plexiglas escape platform $(10$ $\mathrm{cm}$ in diameter) was positioned $2 \mathrm{~cm}$ below the water surface. Extramaze cues surrounding the maze were fixed at specific locations and were visible to the rats while in the maze. Maze performance was recorded by a video camera suspended above the maze and interfaced with a video tracking system (HVS Imaging, Hampton, UK). Rats were temporarily tattooed with a black mark to allow video tracking. Before the spatial working memory task (outlined below), rats were pretrained on a standard place learning protocol until all groups obtained a similar level of performance.

Spatial working memory task. To test spatial working memory, a modified version of a working memory water maze task was used (20,21). During each daily session, rats received four trials consisting of an initial training tria (information trial), and three subsequent retention or choice trials. Times between trials information and choice trials were varied (10 or $100 \mathrm{~min})$. The time between trials 2,3 , and 4 was $30 \mathrm{~s}$. For each subsequent four-trial session, the hidden platform was relocated quasi-randomly to different quadrants and locations of the water maze. Performance was assessed by two measures, mean escape latencies and swim path distances. Escape latencies, swim distances, and swim speeds were analyzed by three-way ANOVA (gender, intermittent hypoxia, delay), followed by Newman-Keuls tests when appropriate.

Tyrosine hydroxylase activity. In vivo tyrosine hydroxylase activity was estimated as previously described (22). Briefly, L-DOPA accumulation was measured after inhibition of dopamine hydroxylase, the enzyme catalyzing the conversion of L-DOPA to DA, using a specific inhibitor, m-hydroxymethylhydrazine (MHBH; Sigma Chemical Co., St. Louis, MO). MHBH was injected intraperitoneally $(100 \mathrm{mg} / \mathrm{kg}$ body weight $) 20 \mathrm{~min}$ before the animals were killed (23). Brains were dissected, and $\mathrm{Cx}, \mathrm{PFC}, \mathrm{CA} 1$, and $\mathrm{CA} 3$ tissues from 5 -mo-old rats exposed to either IH or RA during development ( $n=12$ /group) were stored in $0.1 \mathrm{~N}$ perchloric acid with $0.1 \% \mathrm{wt} / \mathrm{vol}$ EDTA, and frozen a $-70^{\circ} \mathrm{C}$ until analyzed. TH activity in each tissue was expressed as picomoles per milligram protein of L-DOPA formed during $20 \mathrm{~min}$. Neurochemical determination of L-DOPA tissue content was performed using HPLC as previously described, and L-DOPA accumulation was expressed as picomoles per milligram protein per 20 min ( 23-25)

Chemical analyses. PFC and CA1 hippocampal brain tissue samples were obtained from $\mathrm{IH}$ - and normoxia-exposed males and females. Tissues were sonicated in $0.5-1 \mathrm{~mL}$ of $0.1 \mathrm{M} \mathrm{HClO}_{4}$ for $30 \mathrm{~s}$, and centrifuged for $15 \mathrm{~min}$ at $26,000 \mathrm{~g}, 4^{\circ} \mathrm{C}$. Then, a $20-\mathrm{mL}$ supernatant aliquot was injected directly into a HPLC column. For monoamine analyses, a CLC-ODS(M) Shimadzu column (Kyoto, Japan) was used. The mobile phase was $0.163 \mathrm{M}$ citric acid, $\mathrm{pH} 3.0$, containing $0.02 \mathrm{mM}$ EDTA with $0.69 \mathrm{mM}$ sodium octanesulfonic acid as an ion-pairing reagent, $4 \%$ (vol/ $/ \mathrm{vol}$ ) acetonitrile, and $1.7 \%$ (vol/ $/ \mathrm{vol})$ tetrahydrofurane. Norepinephrine, dopamine, 3,4-dihydroxiphenylacetic acid, homovanillic acid; serotonin, and 5-hydroxy-3-indoleacetic acid were electrochemically detected using an amperometric detector (Model L-ECD-6A, Shimadzu), by oxidation on a glass carbon electrode at $0.85 \mathrm{~V}$ in relation to an $\mathrm{Ag}-\mathrm{AgCl}$ reference electrode. The amounts of neurotransmitters and metabolites in the supernatants were calculated by comparing their elution times and peak heights with those of standards. Results are reported as nanograms per gram wet tissue.

Sholl analysis. For morphologic study, rats were anesthetized with sodium pentobarbital and perfused through the ascending aorta with $4 \%$ paraformaldehyde in PBS buffer ( $\mathrm{pH} 7.4$ ) for $45 \mathrm{~min}$. These brains were then postfixed in $10 \%$ formalin in PBS buffer overnight. Blocks of sagittally cut, $10 \%$ formalinfixed rat brain encompassing the prefrontal and piriform cortices were stained with the rapid Golgi method as detailed elsewhere (26). Briefly, the blocks were impregnated using osmium tetroxide plus potassium dichromate, followed by immersion in silver nitrate. After dehydration, the blocks were embedded in nitrocellulose, and coronal sections were cut at $120 \mathrm{~m}$. Camera lucida drawings of the basilar tree were made using a $40 \times$ long-workingdistance oil-immersion lens, a 1.6 Zeiss optivar intermediate lens setting, and a $10 \times$ ocular. For dendritic analyses, six pyramidal neurons in each of prefrontal cortical layers from each animal were randomly selected for assessment $(n=6$ animals for control males and $n=6$ animals for IH-exposed males). Similarly, six pyramidal neurons from the dorsal CA1 region of the hippocampus were also randomly assessed for each animal. A profile for representative neurons in each region of individual animal was generated, with data averaged for each animal. The randomly selected neurons were located in a cortical region starting medially from the midsagittal fissure (but not including the cingulate cortex) and extending laterally to the rhinal fissure. Also, the selected neurons had to meet the following criteria: a soma located in the middle one-third of the thickness of the slide; fully impregnated basilar dendritic tree and spines as ascertained by microscopic observation under high magnification; and no obscuring of branches in the neuropil by glia, blood vessels, or precipitate. The extent and distribution of dendritic branching of these neurons were evaluated by Sholl analysis [method of concentric circles (27)]. Briefly, a transparent overlay of increasingly larger concentric circles at 10 - $\mathrm{m}$ intervals was superimposed on the camera lucida drawing of each basilar tree and the number of dendritic branch intersections (e.g. hits) with each progressively larger circle counted to a distance of $200 \mathrm{~m}$ from the soma of each neuron. The total dendritic length for the basilar tree of each neuron was calculated by tracing camera lucida drawings with a planimeter. Radial tip analysis measured the straight distance between the tips of branches from the center of the cell body. The soma size was measured from the area of a neuron cell body obtained by camera lucida drawings. For spine analysis, only visible flanking dendritic spines were counted along 30-m terminal-tip segments of four total segments for each of five randomly selected neurons per animal under a magnification of $\times 1200$.

\section{RESULTS}

Working memory task. At the time of testing, body weights and brain weights were similar in either IH- or RA-exposed males or females. In males ( $n=12$ /group), exposure to $20 \mathrm{~d} \mathrm{IH}$ during development produced significant long-term working memory deficits at both 10 and 120 min delays when tested 4 mo later (Fig. $1 a$, ANOVA, $p<0.01$ for both latency and pathlength). These differences occurred in the absence of any alterations in T1 latencies or pathlength. In contrast, no effect of IH exposure during development on working memory was observed in female rats on either latency or pathlength measures (Fig. 1b, p- not significant; $n=12$ /group). No significant effect of gender or IH was observed on swim speed, indicating 
$a$

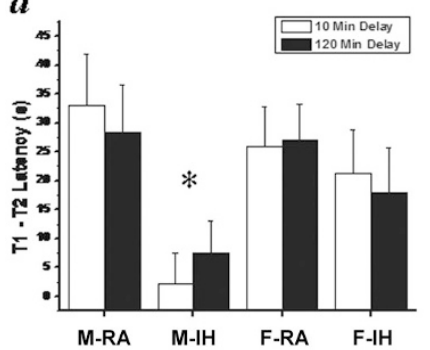

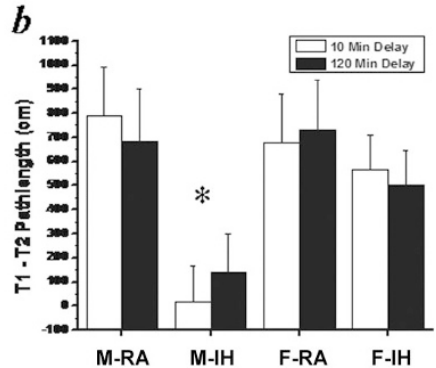

Figure 1. (a) Mean latency (s) savings from trial 1 to trial 2 on a working memory task in 4- to 5-mo-old rats exposed to $20 \mathrm{~d}$ of IH or RA from postnatal d 10 to 30. (b) Mean pathlength $(\mathrm{cm})$ savings from trial 1 to trial 2 on a working memory task in 4- to 5-mo-old rats exposed to $20 \mathrm{~d}$ of IH or RA from postnatal d 10 to 30 . Data are expressed as mean $\pm \mathrm{SE} ; * p<0.01$ vs RA. ( $=12$ /experimental group)

that the observed differences were not due to motor or motivational alterations in our experimental groups (Fig. 2).

Tyrosine hydroxylase activity. Exposure to $20 \mathrm{~d}$ IH during development significantly increased TH activity in the PFC, as measured by DOPA accumulation during $20 \mathrm{~min}$ after inhibition of aromatic amino acid decarboxylase (for males: $\mathrm{IH}, 18.9$ \pm 1.9 versus $\mathrm{RA}, 8.7 \pm 1.5$ pmoles DOPA $/ \mathrm{mg}$ protein; $p<$ $0.01 ; n=12$ /group; Fig. 3). However, no gender differences emerged (NS; Fig. 3). Similarly, TH activity was also increased, albeit to a lesser extent in the $\mathrm{Cx}$ (IH, $7.4 \pm 1.1$ versus RA, $4.7 \pm 0.8$ pmoles DOPA $/ \mathrm{mg}$ protein; $p<0.03 ; n=$ 12/group). Again, no gender differences were present. No significant changes in $\mathrm{TH}$ activity occurred in either CA1 or CA3 hippocampal regions in either male or female rats (Fig. 3).

Monoamine tissue levels. The summary of tissue levels for norepinephrine, dopamine, 3,4-dihydroxiphenylacetic acid, homovanillic acid, serotonin, and 5-hydroxy-3-indoleacetic acid is shown in Table 1. Both males and females exposed to $\mathrm{IH}$ displayed significant increases in norepinephrine levels within the PFC with no gender differences emerging. However, substantial increases in dopamine and 3,4-dihydroxiphenylacetic acid concentrations in the PFC occurred in females exposed to

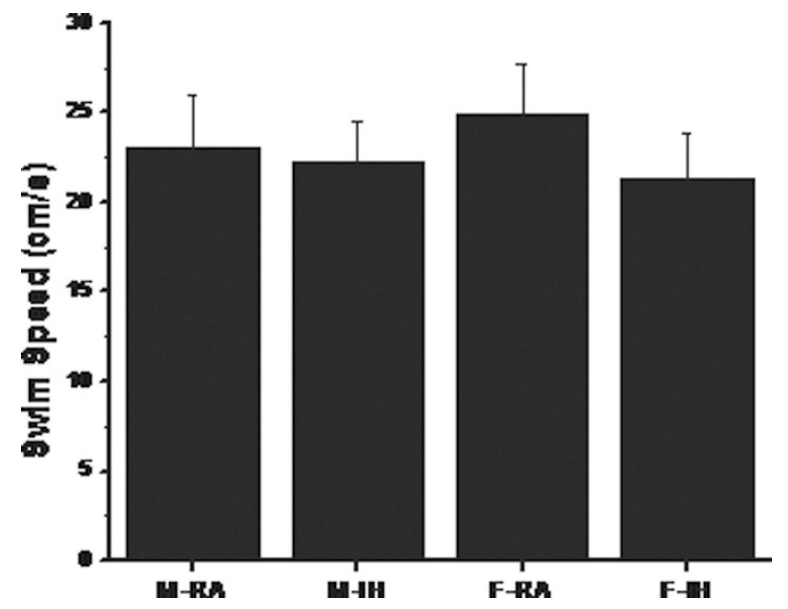

Figure 2. Mean swim speeds $(\mathrm{cm} / \mathrm{s})$ of developing rats exposed to $20 \mathrm{~d}$ of $\mathrm{IH}$ or RA from postnatal d 10 to 30 when tested at $4-5$ mo of age. Data are expressed as mean \pm SE. No significant difference emerged (NS, $n=$ 12/experimental group).

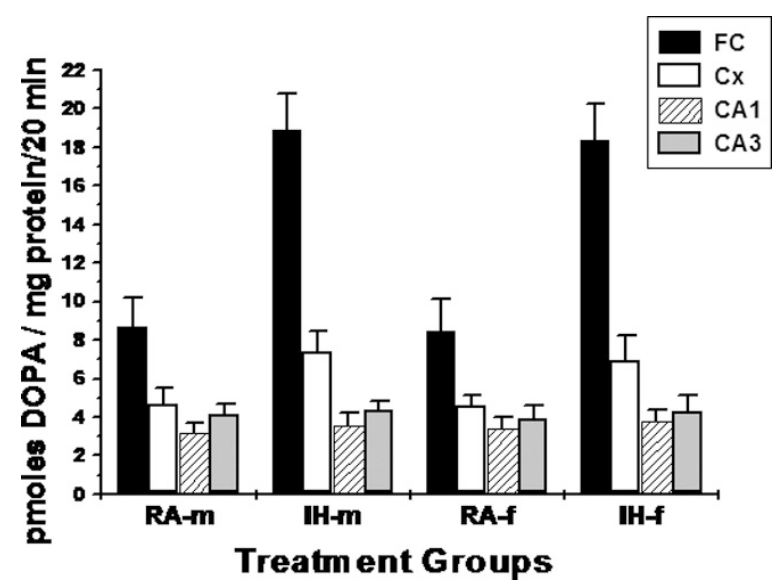

Figure 3. TH activity in neuronal tissue of 5-mo-old rats exposed to $20 \mathrm{~d}$ of IH or RA from postnatal d 10 to 30 . Data are expressed as pm/mg protein/20 $\min \left(\right.$ mean $\pm \mathrm{SE}, n=12$ per experimental group; ${ }^{*} p<0.01$ vs RA). No difference occurred between male $(\mathrm{m})$ and female (f) rats.

$\mathrm{IH}$, but not in males exposed to IH (Table $1 ; p<0.01$ ). No significant differences in the other monoamines were present.

Sholl analysis. Sholl analysis of Golgi-stained neurons revealed a significant decrease in dendritic branching in the layer 5 of the frontal cortex of male rats (Fig $4 a ; p<0.005 ; n=36$ cells per experimental group). No significant differences in dendritic arborization were observed in the CA1 region of the hippocampus (Fig $4 a$; NS; $n=36$ cells per experimental group). Similarly, no differences emerged in the PFC of female rats exposed to either normoxia or intermittent hypoxia. The mean total dendritic length and soma size per neuron were similar across groups. However, the number of dendritic spines tended to be reduced across all layers within the PFC of males, but not of females, exposed to IH ( $\mathrm{F}=2.48 ; p=0.053)$.

\section{DISCUSSION}

This study shows that intermittent hypoxia during a short but highly vulnerable period of postnatal development leads to substantial alterations in working spatial memory in male but not female rats and that these neurobehavioral deficits coincide with reductions in dendritic branching in neurons within the PFC but not within the CA1 region of the hippocampus. Furthermore, gender- and IH-related differences in monoamine brain tissue levels emerged and may account for some of the functional deficits observed in male rats.

Before we discuss the potential implications of our findings, some methodological issues deserve comment. The intermittent hypoxia protocol used in this study was developed such as to elicit arterial $\mathrm{PO}_{2}$ changes that would mimic the duration and magnitude of oxyhemoglobin desaturations occurring during apneic events in patients with sleep-disordered breathing (8). The cycle frequency used herein, however, would correspond to a relatively high degree of disease severity, and, therefore, we cannot infer on the potential consequences of milder conditions. In addition, the overall duration of exposure was selected to correspond to the period of susceptibility to IH that we previously identified (18). Thus, it remains unclear whether exposures of shorter duration are associated with complete recovery or with similar long-term effects to those found in the 
Table 1. Effect of intermittent hypoxia during development on the concentrations of monoamines and metabolites in the hippocampus and pre-frontal cortex of male $(M)$ and female $(F)$ rats

\begin{tabular}{|c|c|c|c|c|c|c|c|c|c|c|c|c|c|c|c|c|c|}
\hline & \multicolumn{3}{|c|}{ NE } & \multicolumn{2}{|r|}{$\mathrm{DA}$} & \multicolumn{3}{|c|}{ DOPAC } & \multicolumn{3}{|c|}{ HVA } & \multicolumn{3}{|c|}{$5-\mathrm{HT}$} & \multicolumn{3}{|c|}{ 5-HIAA } \\
\hline & M & $\mathrm{F}$ & $\begin{array}{c}p \\
\text { value }\end{array}$ & M & $\begin{array}{c}p \\
\text { value }\end{array}$ & M & $\mathrm{F}$ & $\begin{array}{c}p \\
\text { value }\end{array}$ & M & $\mathrm{F}$ & $\begin{array}{c}p \\
\text { value }\end{array}$ & M & $\mathrm{F}$ & $\begin{array}{c}p \\
\text { value }\end{array}$ & M & $\mathrm{F}$ & $\begin{array}{c}p \\
\text { value }\end{array}$ \\
\hline \multicolumn{18}{|c|}{ PFC } \\
\hline RA & $233 \pm 27$ & $245 \pm 29$ & NS & $46 \pm 5$ & $48 \pm 6$ & $34 \pm 3$ & $38 \pm 4$ & NS & $112 \pm 17$ & $113 \pm 18$ & NS & $255 \pm 39$ & $262 \pm 41$ & NS & $587 \pm 65$ & $576 \pm 68$ & NS \\
\hline $\mathrm{IH}$ & $286 \pm 31 *$ & $305 \pm 32 *$ & NS & $48 \pm 6$ & $69 \pm 7 *<0.01$ & $52 \pm 4$ & $79 \pm 5^{*}$ & $<0.03$ & $122 \pm 16$ & $124 \pm 17$ & NS & $248 \pm 40$ & $256 \pm 38$ & NS & $601 \pm 71$ & $599 \pm 70$ & NS \\
\hline \multicolumn{18}{|c|}{ CA1Hippocampus } \\
\hline RA & $221 \pm 32$ & $231 \pm 33$ & NS & $43 \pm 6$ & $41 \pm 6$ & $44 \pm 5$ & $48 \pm 6$ & NS & $104 \pm 15$ & $106 \pm 13$ & NS & $265 \pm 42$ & $276 \pm 45$ & NS & $565 \pm 64$ & $549 \pm 58$ & NS \\
\hline $\mathrm{IH}$ & $228 \pm 29$ & $236 \pm 34$ & NS & $38 \pm 5$ & $39 \pm 6$ & $48 \pm 6$ & $50 \pm 6$ & NS & $109 \pm 17$ & $113 \pm 16$ & NS & $276 \pm 46$ & $281 \pm 46$ & NS & $578 \pm 70$ & $565 \pm 67$ & NS \\
\hline
\end{tabular}

Data are reported as mean $\pm \mathrm{SE}$ ng/g wet tissue. NE, norepinephrine; DA, dopamine; DOPAC, 3,4-dihydroxiphenylacetic acid; HVA, homovanillic acid; 5-HT, serotonin; 5-HIAA, 5-hydroxy-3-indoleacetic acid.

$* p<0.01 \mathrm{RA}$ vs IH.
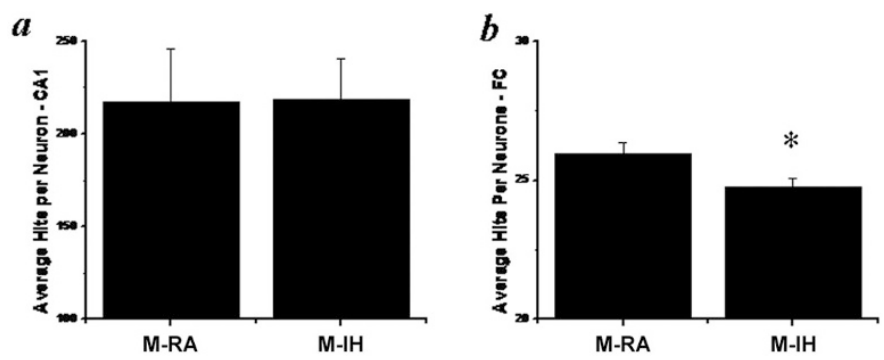

Figure 4. (a) Average number of hits per neuron (Sholl ring crosses) in the hippocampal CA1 region (FC) of 5-mo-old male rats exposed to $20 \mathrm{~d}$ of $\mathrm{IH}$ or RA from postnatal d 10-30. No differences were present among female rats. (b) Average number of hits per neuron (Sholl ring crosses) in the frontal cortex (FC) of 5-mo-old male rats exposed to $20 \mathrm{~d}$ of IH or RA from postnatal d 10 to 30 . Data are expressed as mean $\pm \mathrm{SE} ; * p<0.01$ vs RA.

present study. In other words, systematic exploration of the relative contributions and potential interactions between hypoxic stimulus severity, cycle frequency, and overall exposure duration will be critically needed in future experiments to better define the conditions and thresholds that lead to either complete reversibility or to persistent neural dysfunction.

Precautions were taken to standardize all aspects of animal handling including time of weaning, allocation to genderspecific cages, and circadian timing of the water maze routines. We further allowed for several training days in excess of those needed to reach asymptotic learning in the spatial reference task to ascertain that performance in this task was similar in the two treatment groups. Nevertheless, the paradigm selected for working memory assessment is one of relative complexity, since it requires the coordinated action of both frontal and temporal lobe structures (28), and such complexity may have exacerbated the differences between IH- and RA-exposed groups. Notwithstanding such concerns, it should be emphasized that the inclusion of a short latency delay in the experimental design was based on these very same considerations and aimed to verify the reproducibility of the findings using the longer latency period.

The long-term impact of hypoxia during development on brain function during adulthood has only been summarily explored thus far. Grosjean and colleagues (29) have shown that a single 20 -min exposure to severe hypoxia/anoxia leads to long-term slowing in the ability to acquire a variety of neurobehavioral tasks. In another recent study, Simonova and colleagues (30) exposed newborn rats to intermittent hypobaric hypoxia for the first $19 \mathrm{~d}$ of life. The escape latencies from a spatial memory task using the Morris water maze were longer in the rats exposed to $\mathrm{IH}$ both upon completion of the exposure and approximately 3 mo later. These functional deficits were associated with decreased staining for neurofilament immunoreactivity, astrocytes (GFAP), and oligodendrocytes (RIP) in the hippocampus and somatosensory and motor cortices in the younger animals and only minimal changes in these regions upon reaching adulthood (30). Similar findings have been reported using other hypoxic paradigms during early postnatal life (31-33). For example, Nyakas and colleagues (34) have conclusively demonstrated that application of hypoxia or hypoxic-like treatments leads to substantial effects in the architectural development of specific neural regions (e.g. striatum, cortex, and hippocampus), and that these anatomical changes are accompanied by changes in the expression of recognized neurotransmitters (i.e. cholinergic and dopaminergic pathways) as well as by performance deficits in the underlying neurocognitive substrates. In our previous work, we specifically showed that IH leads to acute deterioration in spatial reference task acquisition and retention and that, in male rats, these deficits are further associated with increased locomotor activity (9). Conversely, application of the same IH paradigm as the one used herein during gestation did not reveal any significant alteration in the ability of offspring to acquire a spatial reference task at either $30 \mathrm{~d}$ or $120 \mathrm{~d}$ of life, despite lifelong modification of the neural correlates of ventilatory control (12). The overall number of iterations required to achieve similar performances in the spatial reference task was higher in IH-treated adult males compared with their corresponding normoxic controls. This suggests that although the ability to acquire the task is somewhat disrupted as a long-term consequence of IH, overall learning can be achieved, thereby suggesting that the major problem underlying the learning process may be the result of prefrontal cortical dysfunction rather than represent hippocampal dysfunction per se. The latter dysfunction is further less likely to be present when considering the normal dendritic arborization found in randomly selected neurons from the CA1 region in male rats. In contrast, the reduced dendritic branching found in the male rats in frontal cortical neurons lends credence to the assumption that the prefrontal structures underlying executive tasks such as those required for adequate spatial working memory, are particularly susceptible to disruption by $\mathrm{IH}$, and that the structural 
changes identified by the unbiased and blinded Sholl analyses are associated with decreased functions that rely on intact prefrontal cortex integrity.

Gender differences were clearly present in this study and displayed improved functional outcomes for female rats compared with their male littermates. The mechanisms mediating such disparities between the functional and structural abnormalities found among male rats and their absence in female rats are unknown and clearly intriguing, considering the absence of gender-related differences in the susceptibility to IH-induced apoptosis (18). Interestingly, we found alterations in $\mathrm{TH}$ activity after $\mathrm{IH}$, but these were not selectively represented in the male rats, and, in fact, similar changes occurred in both males and females. However, dopamine levels were significantly higher in females within the PFC compared with males, whereas the increases in norepinephrine in this region were similar across genders. Based on such findings, we can postulate that disruption of dopaminergic pathways, rather than norepinephrine pathways, as a consequence of $\mathrm{IH}$, may be a direct causal mechanism responsible for the selective genderdependent effect of $\mathrm{IH}$ on working memory. Alternatively, gender differences in the neuroanatomy, dopaminergic innervation of the striatum, the $\mathrm{PFC}$, and/or changes in catecholaminergic receptors could be involved in the observed sexual dimorphism (34-36). Of note, similar changes in components of the dopamine neurotransmitter regulatory cascade were identified by Decker and colleagues (19) within selective regions such as the striatum following perinatal intermittent hypoxia. Although the IH paradigm and overall study design markedly differed between Decker and colleagues and the present study, the striking similarities in neurobehavioral vulnerability and disruption of TH-related pathways in these two studies suggests that a potential mechanistic relationship between the alterations in neurotransmitter and in function. Thus, we cannot exclude the possibility that hormonal gender-related receptors may differentially affect the function of the dopaminergic pathways in the affected brain regions, such that either their intrinsic neurotransmitter pathway putative function is restored in the females or that the recovery-plasticity capability of such neurons is enhanced by the presence of estrogen receptors and their activity $(35,38)$. While these assumptions will have to await more specifically targeted studies on this issue and are clearly beyond the scope of the present work, it is intriguing, albeit not totally unexpected (39), that exposures to IH before pubertal onset may yield gender-related differences.

In summary, we have shown that application during a restricted developmental period of intermittent hypoxic patterns that mimic the oscillatory behavior of oxygen saturation occurring in patients with obstructive sleep apnea leads to altered regulation of tyrosine hydroxylase activity in cortical regions, and also to gender-selective disruption of working memory performance during adulthood. The latter findings may be accounted at least in part by concomitant decreases in dendritic arborization in frontal cortical neurons, and are reminiscent of the reduced school performances found among older children who snored during early childhood (40).

\section{REFERENCES}

1. Partinen M, Telakivi T 1992 Epidemiology of obstructive sleep apnea syndrome. Sleep 15:S1-S4

2. Redline S, Young T 1993 Epidemiology and natural history of obstructive sleep apnea. Ear Nose Throat J 72:20-26

3. Young T, Peppard PE, Gottlieb DJ 2002 Epidemiology of obstructive sleep apnea: a population health perspective. Am J Respir Crit Care Med 165:1217-1239

4. Kales A, Caldwell AB, Cadieux RJ, Vela-Bueno A, Ruch LG, Mayes SD 1985 Severe obstructive sleep apnea-II: Associated psychopathology and psychosocial consequences. J Chronic Dis 38:427-434

5. Bedard MA, Montplaisir J, Richer F, Rouleau I, Malo J 1991 Obstructive sleep apnea syndrome: pathogenesis of neuropsychological deficits. J Clin Exp Neuropsychol 13:950-964

6. Gozal D 2001 Morbidity of obstructive sleep apnea in children: facts and theory. Sleep Breath 5:35-42

7. Beebe DW, Gozal D 2002 Obstructive sleep apnea and the prefrontal cortex: towards a comprehensive model linking nocturnal upper airway obstruction to daytime cognitive and behavioral deficits. J Sleep Res 11:1-16

8. Gozal D, Daniel JM, Dohanich GP 2001 Behavioral and anatomical correlates of chronic episodic hypoxia during sleep in the rat. J Neurosci 21:2442-2450

9. Row BW, Kheirandish L, Neville JJ, Gozal D 2002 Impaired spatial learning and hyperactivity in developing rats exposed to intermittent hypoxia. Pediatr Res 52:449 453

10. Goldbart A, Cheng ZJ, Brittian KR, Gozal D 2003 Intermittent hypoxia induces time-dependent changes in the protein kinase B signaling pathway in the hippocampal CA1 region of the rat. Neurobiol Dis. 14:440-446.

11. Goldbart A, Row BW, Kheirandish L, Schurr A, Gozal E, Guo SZ, Payne RS, Cheng Z, Brittian KR, Gozal D 2003 Intermittent hypoxic exposure during light phase induces changes in cAMP response element binding protein activity in the rat CA1 hippocampal region: water maze performance correlates. Neuroscience 122:585-590

12. Gozal D, Reeves SR, Row BW, Neville JJ, Guo SZ, Lipton AJ 2003 Respiratory effects of gestational intermittent hypoxia in the developing rat. Am J Respir Crit Care Med 167:1540-1547

13. Gozal D, Row BW, Gozal E, Kheirandish L, Neville JJ, Brittian KR, Sachleben LR Jr, Guo SZ 2003 Temporal aspects of spatial task performance during intermittent hypoxia in the rat: evidence for neurogenesis. Eur J Neurosci 18:2335-2342

14. Gozal D, Row BW, Kheirandish L, Liu R, Guo SZ, Qiang F, Brittian KR 2003 Increased susceptibility to intermittent hypoxia in aging rats: changes in proteasomal activity, neuronal apoptosis and spatial function. J Neurochem 86:1545-1552

15. Li RC, Row BW, Gozal E, Kheirandish L, Fan Q, Brittian KR, Guo SZ, Sachleben LR Jr, Gozal D 2003 Cyclooxygenase 2 and intermittent hypoxia-induced spatial deficits in the rat. Am J Respir Crit Care Med 168:469-475

16. Row BW, Goldbart A, Gozal E, Gozal D 2003 Spatial pre-training attenuates hippocampal impairments in rats exposed to intermittent hypoxia. Neurosci Lett 339:67-71

17. Row BW, Liu R, Xu W, Kheirandish L, Gozal D 2003 Intermittent hypoxia is associated with oxidative stress and spatial learning deficits in the rat. Am J Respir Crit Care Med 167:1548-1553

18. Gozal E, Row BW, Schurr A, Gozal D 2001 Developmental differences in cortical and hippocampal vulnerability to intermittent hypoxia in the rat. Neurosci Lett 305:197-201

19. Decker MJ, Hue GE, Caudle WM, Miller GW, Keating GL, Rye DB 2003 Episodic neonatal hypoxia evokes executive dysfunction and regionally specific alterations in markers of dopamine signaling. Neuroscience 117:417-425

20. Hodges H 1996 Maze procedures: the radial-arm and water maze compared. Brain Res Cogn Brain Res 3:167-181

21. Steele RJ, Morris RG 1999 Delay-dependent impairment of a matching-to-place task with chronic and intrahippocampal infusion of the NMDA-antagonist D-AP5. Hippocampus 9:118-136

22. Mamet J, Peyronnet J, Roux JC, Perrin D, Cottet-Emard JM, Pequignot JM, Lagercrantz H, Dalmaz Y 2002 Long-term prenatal hypoxia alters maturation of adrenal medulla in rat. Pediatr Res 51:207-214

23. Soulage C, Perrin D, Cottet-Emard JM, Pequignot J, Dalmaz Y, Pequignot JM 2004 Central and peripheral changes in catecholamine biosynthesis and turnover in rats after a short period of ozone exposure. Neurochem Int 45:979-986

24. Pepin JL, Levy P, Garcin A, Feuerstein C, Savasta M 1996 Effects of long-term hypoxia on tyrosine hydroxylase protein content in catecholaminergic rat brainstem areas: a quantitative autoradiographic study. Brain Res 733:1-8

25. Roux JC, Pequignot JM, Dumas S, Pascual O, Ghilini G, Pequignot J, Mallet J, Denavit-Saubie M 2000 O2-sensing after carotid chemodenervation: hypoxic ventilatory responsiveness and upregulation of tyrosine hydroxylase mRNA in brainstem catecholaminergic cells. Eur J Neurosci 12:3181-3190

26. Valverde F 1976 Aspects of cortical organization related to the geometry of neurons with intra-cortical axons. J Neurocytol 5:509-529

27. Sholl DA 1953 Dendritic organization in the neurons of the visual and motor cortices of the cat. J Anat 87:387-406

28. Simons JS, Spiers HJ 2003 Prefrontal and medial temporal lobe interactions in long-term memory. Nat Rev Neurosci 4:637-648

29. Grojean S, Schroeder H, Pourie G, Charriaut-Marlangue C, Koziel V, Desor D, Vert P, Daval JL 2003 Histopathological alterations and functional brain deficits after transient hypoxia in the newborn rat pup: a long term follow-up. Neurobiol Dis 14:265-278

30. Simonova Z, Sterbova K, Brozek G, Komarek V, Sykova E 2003 Postnatal hypobaric hypoxia in rats impairs water maze learning and the morphology of neurones and macroglia in cortex and hippocampus. Behav Brain Res 141:195-205 
31. Nyakas C, Markel E, Schuurman T, Luiten PG 1991 Impaired learning and abnormal open-field behaviours of rats after early postnatal anoxia and the beneficial effect of the calcium antagonist nimodipine. Eur J Neurosci 3:168-174

32. Hermans RH, Hunter DE, McGivern RF, Cain CD, Longo LD 1992 Behavioral sequelae in young rats of acute intermittent antenatal hypoxia. Neurotoxicol Terato 14:119-129

33. Nyakas C, Buwalda B, Kramers RJ, Traber J, Luiten PG 1994 Postnatal development of hippocampal and neocortical cholinergic and serotonergic innervation in rat: effects of nitrite-induced prenatal hypoxia and nimodipine treatment. Neuroscience 59:541-559

34. Nyakas C, Buwalda B, Luiten PG 1996 Hypoxia and brain development. Prog Neurobiol 49:1-51.

35. Becker JB. Gender differences in dopaminergic function in striatum and nucleus accumbens. Pharmacol Biochem Behav 64:803-812.
36. Andersen SL, Teicher MH 2000 Sex differences in dopamine receptors and their relevance to ADHD. Neurosci Biobehav Rev 24:137-141

37. Markham JA, Juraska JM 2002 Aging and sex influence the anatomy of the rat anterior cingulate cortex. Neurobiol Aging 23:579-588

38. Stone DJ, Rozovsky I, Morgan TE, Anderson CP, Lopez LM, Shick J, Finch CE 2000 Effects of age on gene expression during estrogen-induced synaptic sprouting in the female rat. Exp Neurol 165:46-57

39. Kellogg CK, Primus RJ, Bitran D 1991 Sexually dimorphic influence of prenatal exposure to diazepam on behavioral responses to environmental challenge and on gamma-aminobutyric acid (GABA)-stimulated chloride uptake in the brain. J Pharmacol Exp Ther 256:259-265

40. Gozal D, Pope DW Jr 2001 Snoring during early childhood and academic performance at ages thirteen to fourteen years. Pediatrics 107:1394-1399 